\title{
SMURF1 promotes the proliferation, migration and invasion of gastric cancer cells
}

\author{
YOUMAO TAO, CAIXIA SUN, TAO ZHANG and YAN SONG \\ Department of Gastrointestinal Colorectal and Anal Surgery, China-Japan Union Hospital, \\ Jilin University, Changchun, Jilin 130033, P.R. China
}

Received January 19, 2017; Accepted July 6, 2017

DOI: $10.3892 /$ or.2017.5825

\begin{abstract}
Smad ubiquitin regulatory factor 1 (SMURF1), a well-known E3 ubiquitin ligase, targets substrate proteins for ubiquitination and proteasomal degradation. Accumulating studies have shown that SMURF1 acts as an oncogenic factor in human malignancies. However, the clinical significance of SMURF1 and its role in gastric cancer (GC) remain unclear. The expression of SMURF1 was detected in 68 cases of GC and corresponding tumor-adjacent specimens. Our results revealed that SMURF1 was prominently overexpressed in GC specimens compared to corresponding tumor-adjacent tissues. Furthermore, increased levels of SMURF1 mRNA were also observed in GC cell lines. Clinicopathological detection ascertained that SMURF1-positive expression was associated with large tumor size, more lymph nodes and distant metastasis as well as advanced tumor-node-metastasis (TNM) stage of GC. Notably, GC patients with SMURF1 positive-expressing tumors exhibited a significant decreased survival. Further experiments illustrated that SMURF1 knockdown significantly inhibited proliferation, migration and invasion of MGC-803 cells, while SMURF1 overexpression prominently promoted these behaviors in SGC-7901 cells. In vivo studies revealed that SMURF1 knockdown markedly inhibited tumor growth and liver metastasis of GC. Mechanically, SMURF1 inversely regulated the expression of DOC-2/DAB2 interactive protein (DAB2IP) in GC tissues and cells. Furthermore, DAB2IP restoration revealed similar effects to SMURF1 knockdown on MGC-803 cells with decreased proliferation, migration and invasion. In addition, the PI3K/Akt pathway and its downstream targets including c-Myc and ZEB1 were potentially involved in the oncogenic role of the SMURF1/DABIP axis. Collectively, the present study revealed the first evidence that SMURF1 can be potentially used as a clinical biomarker and target for novel treatment of human GC.
\end{abstract}

Correspondence to: Dr Yan Song, Department of Gastrointestinal Colorectal and Anal Surgery, China-Japan Union Hospital, Jilin University, 126 Xiantai Street, Changchun, Jilin 130033, P.R. China E-mail: songyan_cjuh@163.com

Key words: SMURF1, tumor growth, invasion, migration, GC

\section{Introduction}

Gastric cancer (GC) represents one of the commonest human malignancies characterized by an adverse clinical outcome (1). Radical surgery may be the only hope for curing $\mathrm{GC}$ in the stage of precursor lesions (2). However, even after surgical resection, radiotherapy and chemotherapy provide little benefit (3). Previous studies revealed that a few molecular markers were correlated with prognosis, but the mechanism of GC remains to be elucidated (4). Therefore, it is essential to recognize oncogenesis-associated biomarkers, which are helpful for developing novel treatment in view of GC.

Smad ubiquitin regulatory factor 1 (SMURF1), a recently identified E3 ubiquitin ligase, targets substrate proteins for ubiquitination and proteasomal degradation (5). Increasing evidence has demonstrated that SMURF1 exerts a promoting role in carcinogenesis by targeting downstream proteins for proteolysis. Epidermal growth factor (EGF)-induced SMURF1 overexpression promoted breast cancer cell migration and invasion by targeting RhoA (6). Tumor necrosis factor receptor-associated factor 4 (TRAF4) was also reported to be a substrate protein of SMURF1 and promoted the migration of breast cancer cells (7). SMURF1 was identified as a potential oncogene and a good candidate as a therapeutic target of pancreatic cancer (PC) $(8,9)$. The overexpression of SMURF1 was observed in human colorectal cancer (CRC), and contributed to tumor progression and poor prognosis (10). Several studies have reported that the upstream regulator of SMURF1, IQ motif containing GTPase activating protein 1 (IQGAP1) promoted the ubiquitination and degradation of TGF- $\beta$ receptor II by facilitating the targeting of SMURF1 to the plasma membrane in hepatic stellate cells (11). Casein kinase-2 interacting protein-1 (CKIP-1) suppressed colon cancer cell growth and migration by inhibiting SMURF1 synthesis and facilitating SMURF1 autodegradation (12). Furthermore, SMURF1 was recognized as a direct target of miR-497 in ovarian cancer cells and exerted a pro-metastatic effect (13). TLX, a highly expressed nuclear receptor, physically interacted with and stabilized SMURF1 in glioblastoma (14). In addition, SMURF1 facilitated T98G cell proliferation and migration by regulation of DOC-2/DAB2 interactive protein (DAB2IP) (15). However, the clinical significance of SMURF1 and its role in human GC remain poorly elucidated. 
The present study revealed that SMURF1 overexpression predicted malignant clinical features and decreased survival. We also demonstrated that SMURF1 promoted GC cell growth and metastasis possibly by suppressing DAB2IP. In conclusion, the present study revealed the first evidence that SMURF1 is a clinical biomarker, and recognized as a potential therapeutic target for GC.

\section{Materials and methods}

Patients. Sixty-eight cases of GC and corresponding tumor-adjacent specimens were obtained from the Department of Gastrointestinal Colorectal and Anal Surgery, China-Japan Union Hospital, Jilin University. Tissue specimens were conserved in liquid nitrogen for qRT-PCR or $10 \%$ formalin for IHC until use. Informed consent from patients was obtained before the use of all samples. All the clinicopathological information of patients is presented in Table I. The Ethics Committee of Jilin University approved the present study according to the Declaration of Helsinki.

Cell culture and transfection. GC-derived cell lines (SGC-7901, MGC-803, MKN-28 and BGC-823) and a normal gastric epithelium cell line (GES-1) were obtained from the Cell Bank of the Shanghai Institute of Cell Biology (Chinese Academy of Medical Sciences, Shanghai, China). The cell lines were cultured in Dulbecco's modified Eagle's medium (DMEM) with 10\% fetal bovine serum (FBS) (Gibco, Grand Island, NY, USA) with antibiotics (Sigma-Aldrich, St. Louis, $\mathrm{MO}, \mathrm{USA}$ ) in a incubator containing a $5 \% \mathrm{CO}_{2}$ humidified atmosphere at $37^{\circ} \mathrm{C}$.

Small hairpin RNA (shRNA) targeting SMURF1 and DAB2IP as well as non-targeting (NT) shRNA were obtained from Santa Cruz Biotechnology (Santa Cruz, CA, USA). pcDNA3.1-SMURF1 and pcDNA3.1-DAB2IP were synthesized and purchased from GeneChem (Shanghai, China). All vectors were transferred into cells using Lipofectamine 2000 (Thermo Fisher Scientific, Waltham, MA, USA) on the basis of the manufacturer's recommendations.

Immunohistochemistry (IHC). The tissues that were previously formalin-fixed and paraffin-embedded were sliced into $4 \mu \mathrm{m}$ sections, and underwent deparaffination and then rehydration. Antigen retrieval, suppression of endogenous peroxidase activity and $10 \%$ skim milk blocking were performed before primary antibody incubation. The SMURF1 (Abcam, Cambridge, MA, USA) and DAB2IP (Santa Cruz Biotechnology) antibodies were used as primary antibodies overnight at $4^{\circ} \mathrm{C}$. The slides were subsequently incubated with a peroxidase-conjugated secondary antibody (ZSGB-BIO, Beijing, China) for $90 \mathrm{~min}$, and a peroxidase-labeled polymer, DAB solution was used for signal development for $5 \mathrm{~min}$. The sections were counterstained with hematoxylin followed by dehydration and mounting. Staining intensity was scored as: no staining, 0 ; weak staining, 1 ; moderate staining, 2; and strong staining, 3. Staining quantity was graded as: $<25 \%, 1 ; 25-75 \%, 2$; and $>75 \%, 3$. IHC score was manually confirmed by two independent experienced pathologists using the formula: IHC score = staining intensity x staining quantity. Sections with an IHC score $>1$ were considered SMURF1- and DAB2IP-positive expression.
Table I. Correlation between SMURF1 expression and clinicopathological features in gastric cancer.

\begin{tabular}{|c|c|c|c|c|}
\hline \multirow[b]{2}{*}{ Characteristics } & \multirow[b]{2}{*}{ Total } & \multicolumn{2}{|c|}{ SMURF1 expression } & \multirow[b]{2}{*}{ P-value } \\
\hline & & $\begin{array}{c}\text { Positive } \\
(48)\end{array}$ & $\begin{array}{l}\text { Negative } \\
\text { (20) }\end{array}$ & \\
\hline Age (years) & & & & 0.800 \\
\hline$<65$ & 29 & 20 & 9 & \\
\hline$\geq 65$ & 39 & 28 & 11 & \\
\hline Sex & & & & 0.485 \\
\hline Male & 53 & 39 & 14 & \\
\hline Female & 15 & 9 & 6 & \\
\hline $\begin{array}{l}\text { Tumor } \\
\text { differentiation }\end{array}$ & & & & 0.198 \\
\hline $\mathrm{I}, \mathrm{II}$ & 32 & 25 & 7 & \\
\hline III,IV & 36 & 23 & 13 & \\
\hline Size $(\mathrm{cm})$ & & & & $0.014^{\mathrm{a}}$ \\
\hline$<5$ & 32 & 18 & 14 & \\
\hline$\geq 5$ & 36 & 30 & 6 & \\
\hline Invasive depth & & & & 0.146 \\
\hline $\begin{array}{l}\text { Mucosa to } \\
\text { muscularis } \\
\text { propria }\end{array}$ & 9 & 4 & 5 & \\
\hline $\begin{array}{l}\text { Adventitia to } \\
\text { adjacent structure }\end{array}$ & 59 & 44 & 15 & \\
\hline $\begin{array}{l}\text { Lymph node } \\
\text { metastasis } \\
\text { (regions) }\end{array}$ & & & & $0.016^{\mathrm{a}}$ \\
\hline$\leq 2$ & 29 & 16 & 13 & \\
\hline$>2$ & 39 & 32 & 7 & \\
\hline Distant metastasis & & & & $0.044^{\mathrm{a}}$ \\
\hline No & 52 & 33 & 19 & \\
\hline Yes & 16 & 15 & 1 & \\
\hline Venous infiltration & & & & 0.065 \\
\hline Absent & 51 & 33 & 18 & \\
\hline Present & 17 & 15 & 2 & \\
\hline TNM stage & & & & $0.042^{\mathrm{a}}$ \\
\hline I,II & 28 & 16 & 12 & \\
\hline III,IV & 40 & 32 & 8 & \\
\hline
\end{tabular}

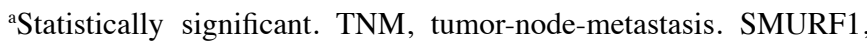
Smad ubiquitin regulatory factor 1 .

Quantitative real-time polymerase chain reaction ( $q R T-P C R)$. Total RNA was drawn using TRIzol (Invitrogen, Carlsbad, CA, USA). The first strand cDNA was compounded using a TIANScript RT kit (Tiangen Biotech, Beijing, China). The expression of SMURF1 mRNA was detected using the ABI 7300 system (Applied Biosystems, Foster City, CA, USA). GAPDH was employed as the internal control. The primers used for target genes were purchased from Sangon Biotech (Shanghai, China). 
Proliferation assay. For cell proliferation, GC cells that were treated with corresponding vectors were seeded into 96-well plates $\left(1.5 \times 10^{3}\right.$ cells/well). After transfection at $24,48,72$ and $96 \mathrm{~h}$, the cell proliferation assay was performed by addition of $10 \mu \mathrm{l}$ Cell Counting Kit-8 (CCK-8) solution (Beyotime, Shanghai, China) to each well, followed by incubation at $37^{\circ} \mathrm{C}$ for $2 \mathrm{~h}$. The absorbance was assessed at a wavelength of $490 \mathrm{~nm}$ using a microplate reader (FlexStation III ROM V2.1.28; Molecular Devices, Sunnyvale, CA, USA).

Wound healing assay. GC cells transduced with corresponding vectors were seeded into 6-well plates to form a single confluent cell layer. The wounds were made with $100 \mu \mathrm{l}$ tips in the confluent cell layer. After wound scratching at 0 and $24 \mathrm{~h}$, the width of the wound was photographed using a phase-contrast microscope.

Transwell invasion assay. We determined the invasion capacities of GC cells using Transwell chambers of pore size 8- $\mu \mathrm{m}$ (Corning Costar, Cambridge, MA, USA). Twenty-four hours after transduction, $5 \times 10^{4}$ cells were cultured in the 1:9 diluted Matrigel-coated (BD Biosciences, Franklin Lakes, NJ, USA) upper chamber with $250 \mu \mathrm{l}$ of serum-free DMEM, while $700 \mu 1$ DMEM with $10 \%$ FBS were added in the lower chamber. After $24 \mathrm{~h}$, we fixed the cells with paraformaldehyde, and the cells in upper chamber were removed. Cells in the lower chamber were subsequently stained using $0.1 \%$ crystal violet solution and photographed.

Experimental mouse model. The in vivo growth ability of GC cells was examined using the subcutaneous implantation nude mouse model. MGC-803 cells transfected with NT-shRNA or SMURF1-shRNA were subcutaneously injected into the left flank of nude mice. After 4 weeks, the subcutaneous tumors were finally resected and subjected to weight assessments. A liver metastasis assay in nude mice was performed using the subcapsular splenic injection model in which the MGC-803 cells were injected to the spleen subcapsular. Nine weeks after splenic injection, all mice were euthanized, and the livers were obtained. Furthermore, analysis of micrometastasis was assessed on the left lateral lobe of the liver, that was fixed and cut sagittally into 4 parts, paraffin-embedded, sectioned and stained for hematoxylin and eosin (H\&E) (16). The protocol for these animal experiments was approved by the Ethics Review Committee of Jilin University.

Western blotting. Cancer cells were dissociated in RIPA lysis buffer (P0013D) and PMSF (ST506) (both from Beyotime, Haimen, China). Split products were centrifuged at $12,000 \mathrm{rpm}$, and then supernatants were gathered. A Bradford protein assay kit (P0006) (Beyotime) was used to analyze the protein concentration, and proteins were loaded onto $10 \%$ SDS-PAGE. Then proteins after separation were transferred onto polyvinylidene fluoride (PVDF) membranes (Sigma-Aldrich). Then, the PVDF membranes were obstructed with 5\% skim milk (GuangMing, Shanghai, China) and incubated with SMURF1 (Abcam), DAB2IP (Santa Cruz Biotechnology), p-Akt (Ser473), Akt, c-Myc or ZEB1 (all from Cell Signaling Technology, Beverly, MA, USA) primary antibodies at $4^{\circ} \mathrm{C}$ overnight. Then, the specimens were incubated with a secondary antibody conjugated with HRP (Cell Signaling Technology). Signals were detected using an HRP chemiluminescent kit (Thermo Fisher Scientific) and an optional CCD camera as well as an image processing system (Bio-Rad, Hercules, CA, USA). GAPDH (G8140; US Biological, Swampscott, MA, USA) was used as a loading control.

Statistical analysis. Data were presented as the mean \pm SEM and analyzed by GraphPad Prism 5 software (GraphPad Software, Inc., San Diego, CA, USA). Chi-squared test was employed to explore the association between two variables. The Student's t-test and ANOVA were carried out to analyze continuous variables. Survival analysis was performed using Kaplan-Meier's method and the log-rank test. A P-value $<0.05$ was considered to have statistical significance.

\section{Results}

SMURF1 overexpression is a clinical biomarker of GCs. Sixty-eight samples of GC and corresponding tumor-adjacent specimens were detected by IHC for SMURF1 staining. Sections with an IHC score $>1$ were considered as positive expression of SMURF1. GC tissue samples, 70.59\% (48/68) exhibited positive staining of SMURF1, while SMURF1 signal were detected in only $47.06 \%$ (32/68) samples of tumor-adjacent specimens ( $\mathrm{P}=0.005$; Fig. 1). Next, qRT-PCR further demonstrated that the levels of SMURF1 mRNA in GC tissues was upregulated compared with corresponding tumor-adjacent tissues $(\mathrm{P}<0.05$; Fig. 2A). Moreover, the levels of SMURF1 mRNA expression in GC cell lines (SGC-7901, MGC-803, MKN-28 and BGC-823) were significantly increased when compared to the normal gastric epithelium cell line (GES-1) $(\mathrm{P}<0.05$, respectively; Fig. 2B). In addition, positive expression of SMURF1 in GC patients was correlated with large-sized tumors, more lymph node metastasis and distant metastasis as well as tumor-node-metastasis $(\mathrm{TNM})$ grade $(\mathrm{P}<0.05$, respectively; Table I). Survival analysis revealed that GC patients with SMURF1-positive expression exhibited a prominent decreased 5-year overall and disease-free survival $(\mathrm{P}<0.05$, respectively; Fig. 2C and D). Thus, SMURF1 may be a potential prognostic biomarker in GC.

SMURF1 regulates proliferation and mobility of GC cells. Since SMURF1 was overexpressed in GC, we speculated that the biological functions of SMURF1 may participate in controlling cell proliferation and metastasis. To verify this hypothesis, SMURF1 expression was silenced by a specific shRNA in MGC-803 cells ( $\mathrm{P}<0.05$; Fig. 3A). CCK-8 assays were used to analyze the effect of SMURF1 silencing on proliferation of GC cells. We found that silencing of SMURF1 suppressed MGC-803 cell proliferation as compared with control cells $(\mathrm{P}<0.05$; Fig. 3B). To disclose the potential role of SMURF1 in the metastasis of GC, we analyzed the migration and invasion of GC cells using wound healing and Transwell invasion assays. The results revealed that SMURF1 knockdown caused a prominent decrease in the migratory and invasive abilities of MGC-803 cells compared to the control cells $(\mathrm{P}<0.05$, respectively; Fig. 3C and D). Next, SGC-7901 cells were transduced with an empty vector (EV) and pcDNA3.1-SMURF1, respectively. 

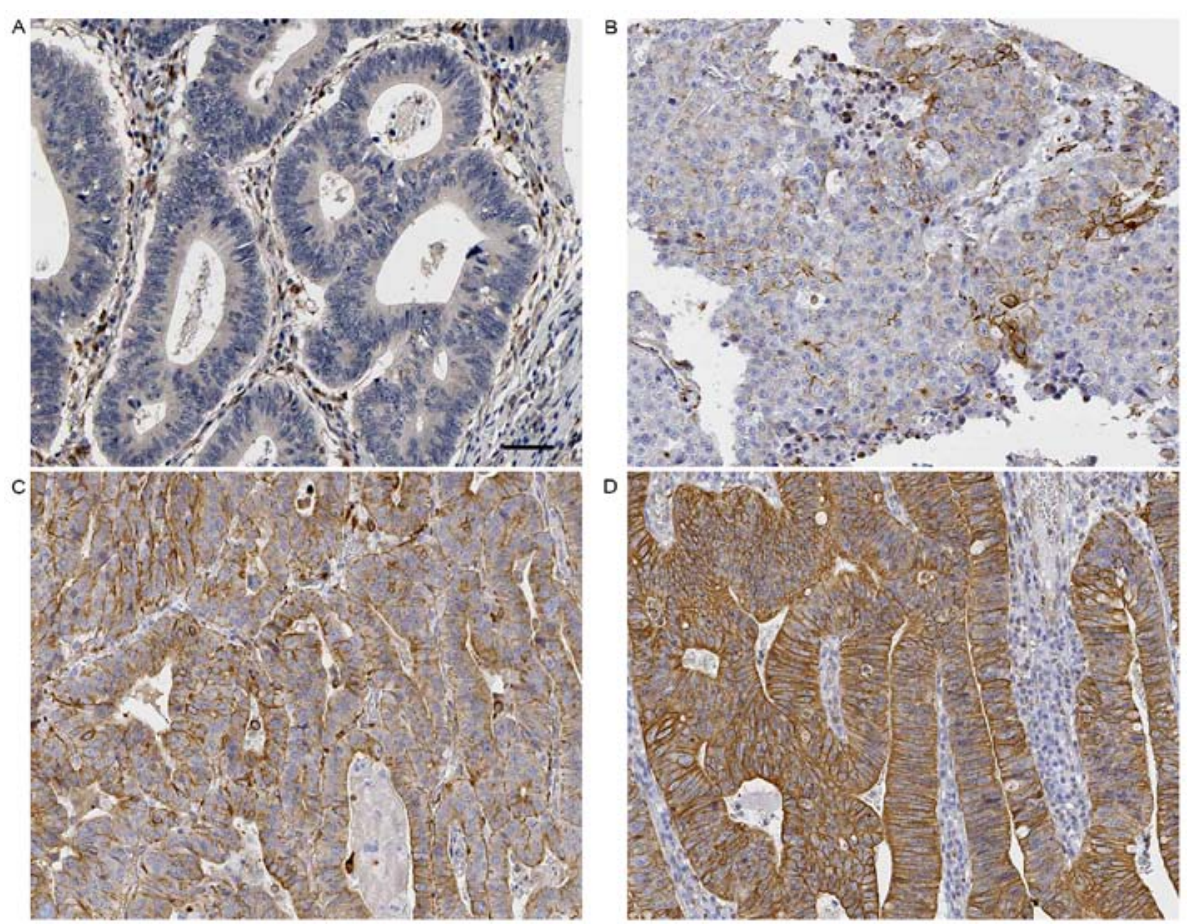

Figure 1. Immunohistochemistry of SMURF1 protein in GC. Representative immunohistochemical staining showed (A) negative, (B) low, (C) medium and (D) high expression of SMURF1 in GC tissues. Scale bar, $50 \mu \mathrm{m}$. SMURF1, smad ubiquitin regulatory factor 1; GC gastric cancer.
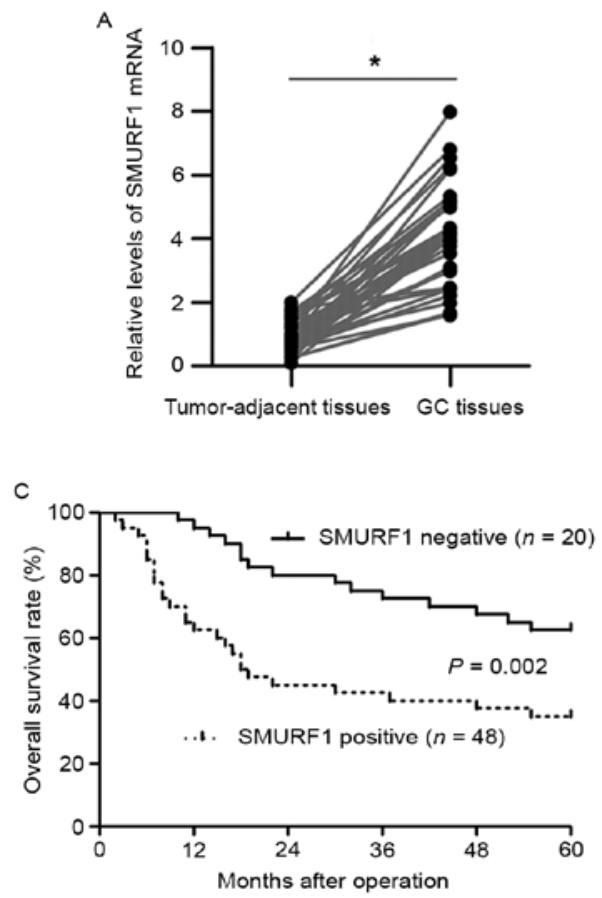
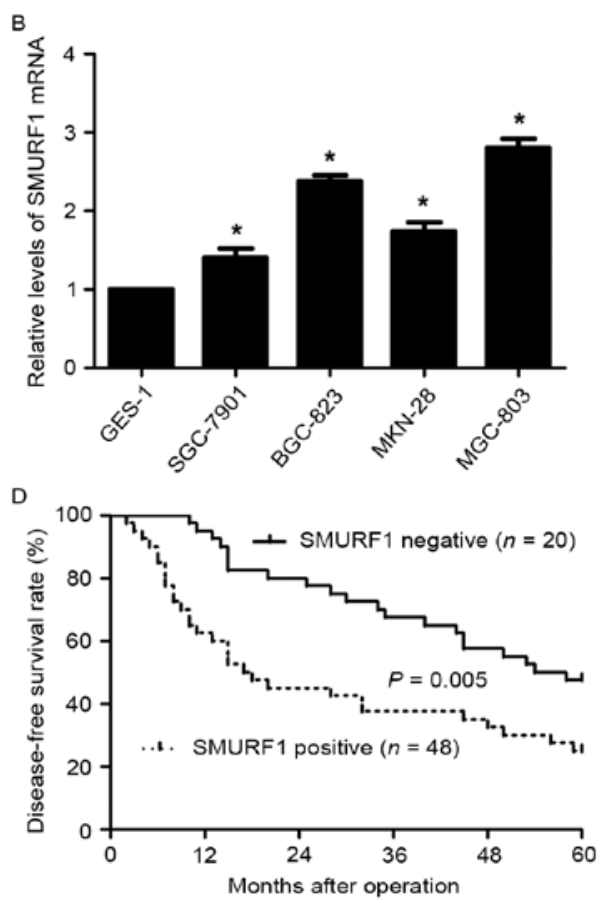

Figure 2. Expression differences of SMURF1 mRNA in GC. (A) The expression of SMURF1 mRNA in GC tissues and corresponding tumor-adjacent specimens; $\mathrm{n}=68,{ }^{*} \mathrm{P}<0.05$. (B) Relative expression of SMURF1 mRNA in GC cell lines (SGC-7901, MGC-803, MKN-28 and BGC-823) and a normal gastric epithelium cell line (GES-1); n=3, ${ }^{*} \mathrm{P}<0.05$. (C and D) SMURF1-positively expressing GC patients exhibited a significant shorter 5-year overall and disease-free survival. SMURF1, smad ubiquitin regulatory factor 1; GC gastric cancer.

SMURF1 overexpression was confirmed by western blotting in SGC-7901 cells $(\mathrm{P}<0.05$; Fig. 4A). Overexpression of SMURF1 notably enhanced the proliferation, migration and invasion of SGC-7901 cells $(\mathrm{P}<0.05$; respectively; Fig. 4B-D). Collectively, all the results demonstrated that SMURF1 can markedly inhibit GC cell proliferation and mobility in vitro.
SMURF1 knockdown decreases the growth and metastasis of GC cells in nude mice. To further confirm the effects of SMURF1 on the growth and metastasis of GC cells, a mouse experimental growth or liver metastasis model was constructed using MGC-803 cells via subcutaneous or subcapsular splenic injection. Silencing of SMURF1 markedly decreased the 

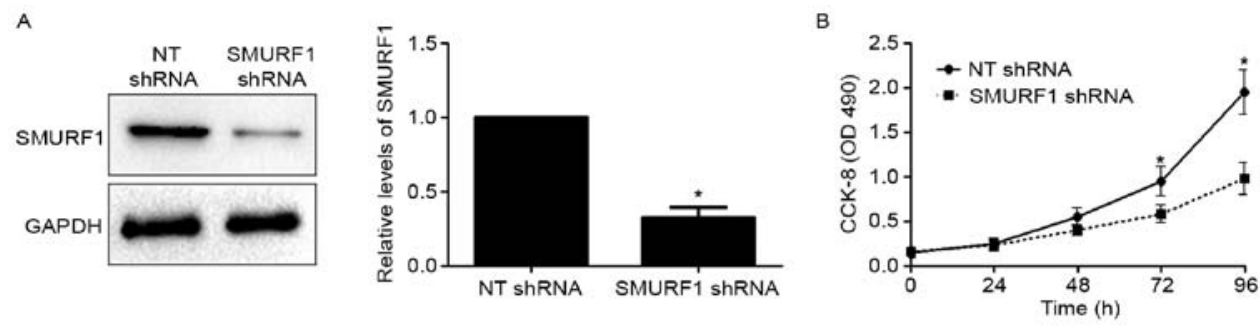

D
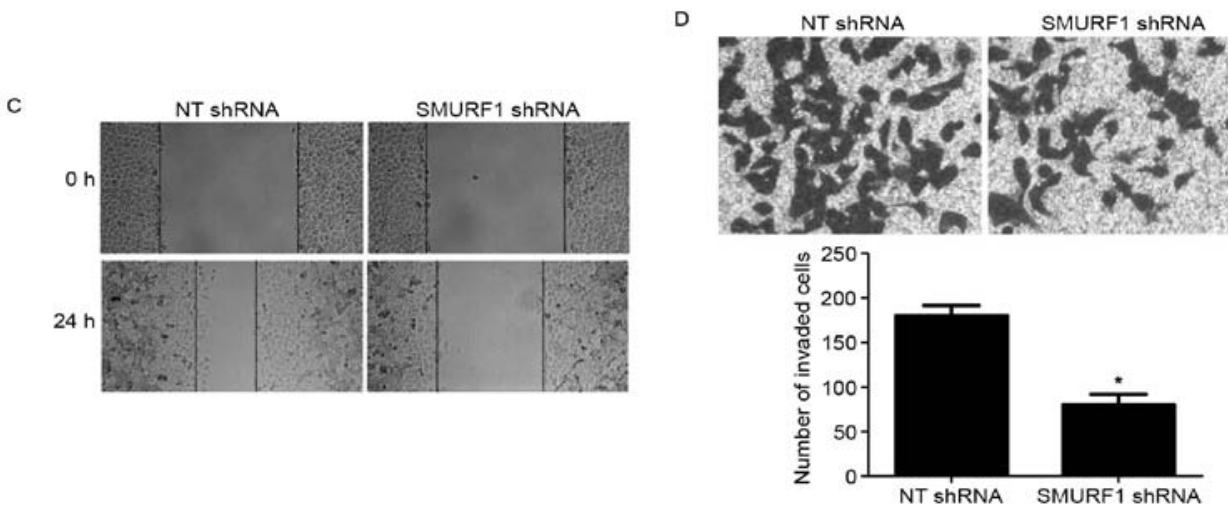

Figure 3. Impact of SMURF1 knockdown on GC cell proliferation and mobility. (A) MGC-803 cells that were transduced with non-targeting (NT) shRNA or SMURF1-shRNA were detected by immunoblotting for SMURF1 expression; $n=3,{ }^{*} \mathrm{P}<0.05$. (B) CCK-8 assays indicated that silencing of SMURF1 suppressed proliferation in MGC- 803 cells; $n=3,{ }^{*} \mathrm{P}<0.05$. (C) Wound healing assays revealed that the migration of MGC-803 cells was suppressed by SMURF1 knockdown. (D) Transwell invasion assays indicated that SMURF knockdown decreased the number of invaded MGC-803 cells; $n=3$, "P<0.05. SMURF1, smad ubiquitin regulatory factor 1 ; GC gastric cancer.
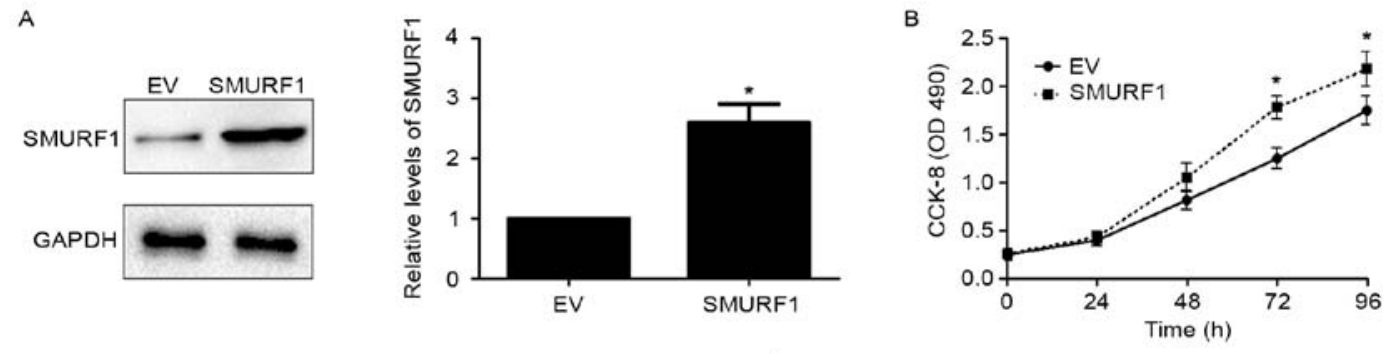

D

EV

SMURF1

C
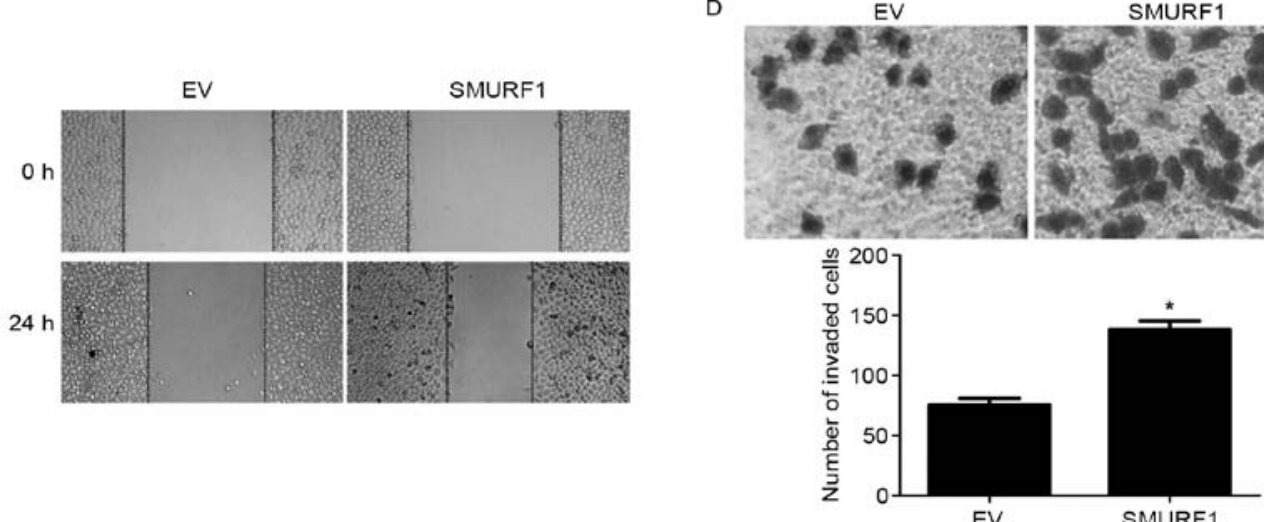

Figure 4. SMURF1 overexpression regulates cell proliferation and mobility in SGCA-7901 cells. (A) SGC-7901 cells that were transduced with pcDNA3.1-SMURF1 or empty vector (EV) were assessed by western blotting for SMURF1 expression; $n=3$, ${ }^{*} \mathrm{P}<0.05$. (B) CCK-8 assays revealed that SMURF1 overexpression facilitated proliferation in SGC-7901 cells; $n=3,{ }^{*} \mathrm{P}<0.05$. (C) Wound healing assays demonstrated that the migration ability of SGC-7901 cells was increased by SMURF1 overexpression. (D) SMURF1 overexpression promoted cell invasion in SGC-7901 cells assessed by Transwell invasion assays; $\mathrm{n}=3,{ }^{*} \mathrm{P}<0.05$. SMURF1, smad ubiquitin regulatory factor 1 ; GC gastric cancer.

subcutaneous growth of GC in nude mice as determined by the tumor weights $(\mathrm{P}<0.05$; Fig. $5 \mathrm{~A})$. In addition, liver metastasis experiments revealed that SMURF1 knockdown notably decreased the number of metastatic nodules in the livers of nude mice $(\mathrm{P}<0.05$; Fig. 5B). Altogether, our data revealed that SMURF1 prominently prohibited GC cell growth and metastasis in vivo.

DAB2IP may be involved in the role of SMURF1. Previous research revealed that SMURF1 promotes cancer cell 

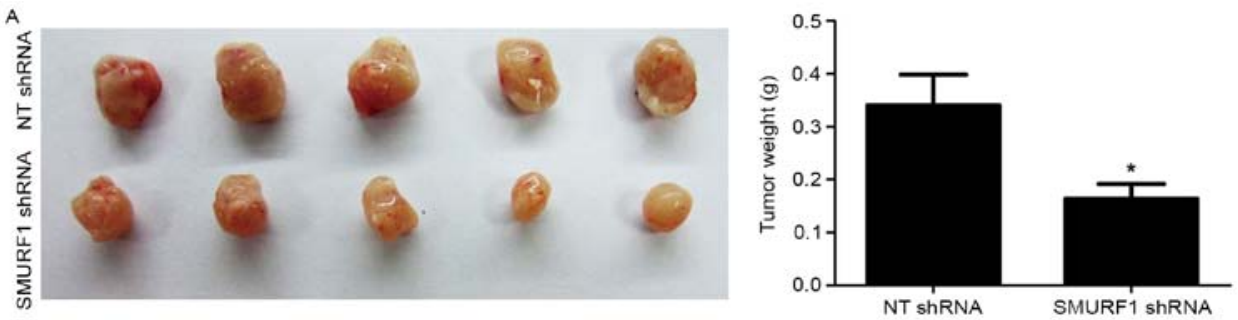

B
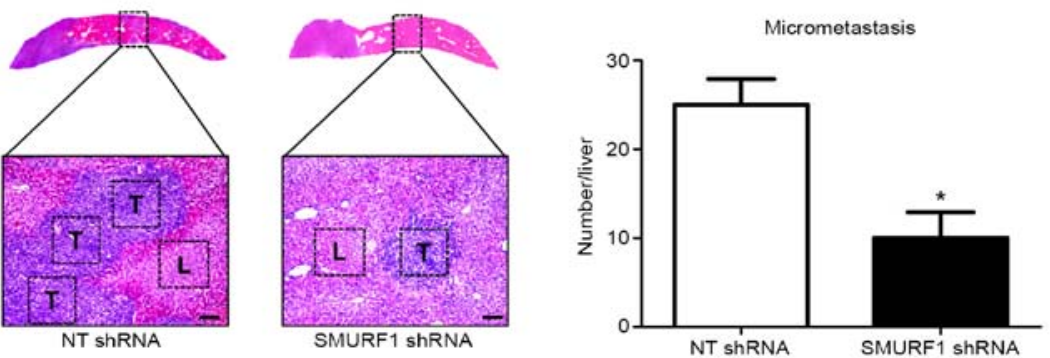

Figure 5. SMURF1 silencing inhibits the growth and liver metastasis of GC in nude mice. (A) MGC-803 cells that were infected with NT-shRNA or SMURF1-shRNA were implanted into nude mice via subcutaneous injection. The tumor weight measurements revealed that SMURF1 knockdown significantly prohibited the growth of GC in vivo; $\mathrm{n}=5,{ }^{*} \mathrm{P}<0.05$. (B) MGC-803 cells that were infected with NT-shRNA or SMURF1-shRNA were injected into the spleen subcapsular. H\&E staining revealed that silencing of SMURF1 significantly decresed liver metastases of MGC-803 cells; $\mathrm{n}=5$, ${ }^{*} \mathrm{P}<0.05$. T, tumor tissue; L, liver tissue. Magnification, x100 and scale bars, $100 \mu \mathrm{m}$. SMURF1, smad ubiquitin regulatory factor 1; GC gastric cancer.
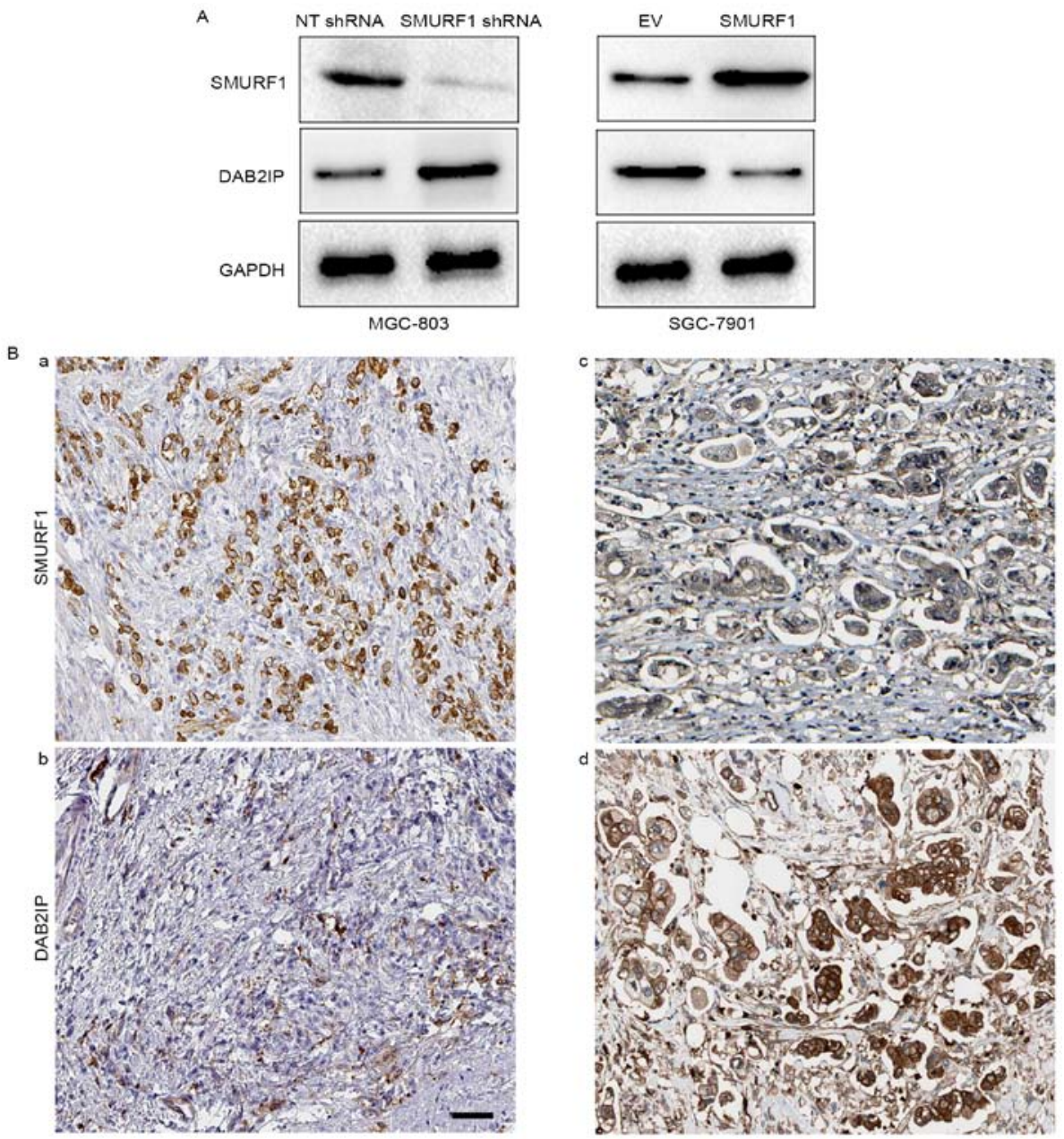

Figure 6. SMURF1 regulates the expression of DAB2IP in GC cells. (A) MGC-803 and SGC-7901 cells that were transfected with corresponding vectors (NT-shRNA and SMURF1-shRNA or EV and pcDNA3.1-SMURF1) were subjected to immunoblotting for SMURF1 and DAB2IP expression. SMURF1 negatively regulated the expression of DAB2IP in GC cells. (B) Representative immunohistochemical staining indicated that highly-expressing SMURF1 GC tissue (a) exhibited weak staining of DAB2IP (b), while strong signals of DAB2IP (d) were observed in low expression of SMURF1 tissues (c). Scale bar, $50 \mu \mathrm{m}$. SMURF1, smad ubiquitin regulatory factor 1; GC gastric cancer DAB2IP, DOC-2/DAB2 interactive protein; EV, empty vector. 

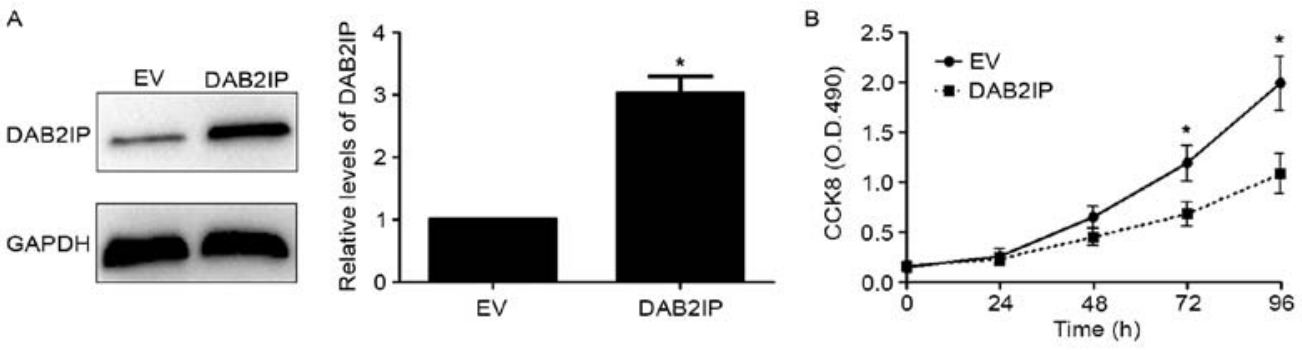

C

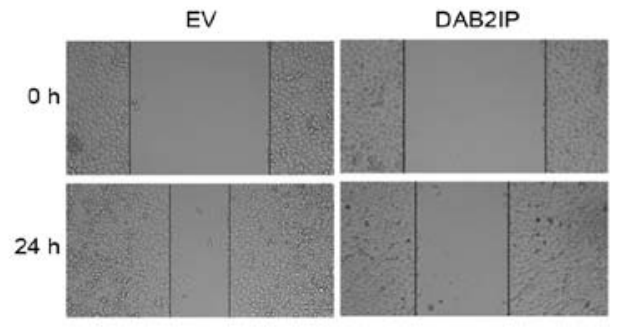

D

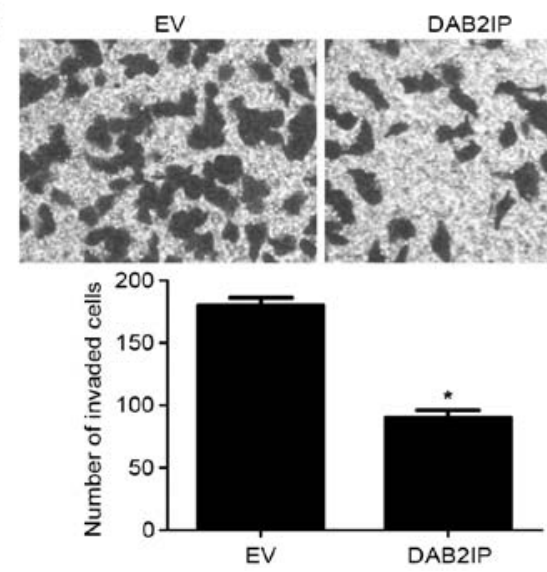

Figure 7. DAB2IP restoration decreases GC cell proliferation and mobility. (A) MGC-803 cells that were transduced with an empty vector (EV) or pcDNA3.1DAB2IP were detected by immunoblotting for DAB2IP expression; $n=3$, ${ }^{*} \mathrm{P}<0.05$. (B) CCK-8 assays indicated that overexpression of DAB2IP suppressed proliferation in MGC-803 cells; $n=3,{ }^{*} \mathrm{P}<0.05$. (C) Wound healing assays revealed that the migration of MGC- 803 cells was suppressed by DAB2IP restoration. (D) Transwell invasion assays indicated that DAB2IP overexpression decreased the number of invaded MGC-803 cells; $n=3$, "P<0.05. DAB2IP, DOC-2/DAB2 interactive protein; GC, gastric cancer.

proliferation and migration by negatively regulating DAB2IP (15). Therefore, we investigated whether DAB2IP was involved in the role of SMURF1 in GC. Notably, we found that the expression of DAB2IP was increased after SMURF1 knockdown in MGC-803 cells (Fig. 6A), while, SMURF1 overexpression led to a decreased level of DAB2IP in SGC-7901 cells (Fig. 6A). Furthermore, DAB2IP staining was performed in GC tissues using IHC. Our data revealed that the positive expression of DAB2IP was observed in $36.76 \%$ (25/68) GC tissue samples. Notably, highly-expressing SMURF1 in GC tissues exhibited weak staining of DAB2IP, while strong signals of DAB2IP were observed in tissues with low expression of SMURF1 (Fig. 6B). Next, DAB2IP was markedly overexpressed by a pcDNA3.1-mediated expression plasmid in MGC-803 cells ( $\mathrm{P}<0.05$; Fig. 7A). Notably, DAB2IP restoration revealed similar effects to SMURF1 knockdown in MGC-803 cells with decreased cell proliferation, migration and invasion $(\mathrm{P}<0.05$, respectively; Fig. 7B-D). Therefore, these results indicate that SMURF1 plays an oncogenic role possibly by suppressing DAB2IP in GC cells.

The PI3K/Akt pathway is potentially involved in the oncogenic role of the SMURF1/DAB2IP axis. DAB2IP has been reported to regulate various signaling pathways including the PI3K/Akt pathway in human cancer (17). Furthermore, the downstream targets of PI3K/Akt pathway such as c-Myc and ZEB1 regulate proliferation, migration and invasion in GC cells $(18,19)$. The results from western blot analysis revealed that SMURF1 knockdown upregulated the level of DAB2IP and subsequently downregulated the expression of phosphorylated Akt as well as c-Myc and ZEB1 in MGC-803 cells (Fig. 8). However, DAB2IP-

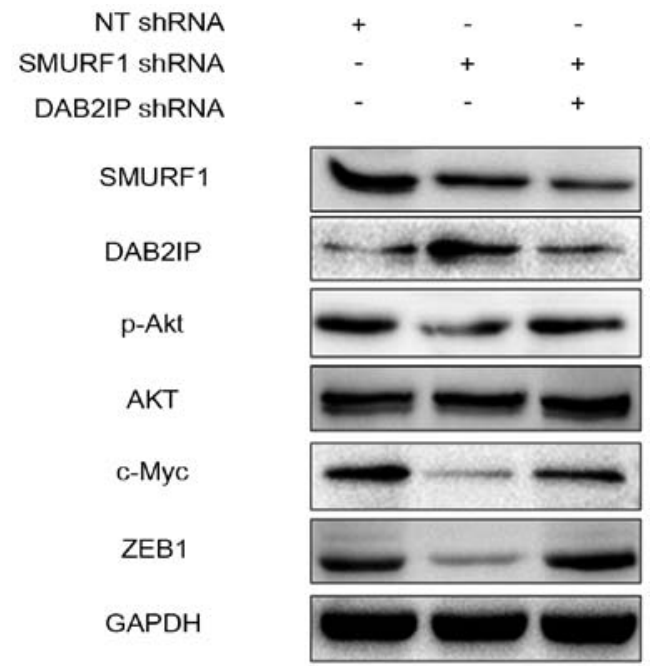

Figure 8. SMURF1 inhibits the activation of the PI3K/Akt pathway in GC cells. MGC-803 cells were transfected with NT shRNA, SMURF1-shRNA or SMURF1-shRNA + DAB2IP-shRNA. Western blot analysis revealed that SMURF1 knockdown upregulated the level of DAB2IP and subsequently downregulated the expression of phosphorylated Akt as well as c-Myc and ZEB1 in MGC-803 cells, while, the effects of SMURF1 knockdown on the activation of the PI3K/Akt pathway were reversed by DAB2IP-silencing. SMURF1, smad ubiquitin regulatory factor 1; GC gastric cancer DAB2IP, DOC-2/DAB2 interactive protein.

silencing abrogated the effects of SMURF1 knockdown on the activation of the PI3K/Akt pathway with increased levels of poshorylated Akt, c-Myc and ZEB1 (Fig. 8). Thus, SMURF1 probably suppresses DAB2IP and subsequently enhances the activation of the PI3K/Akt pathway in GC cells. 


\section{Discussion}

Increasing evidence has demonstrated that SMURF1 participates in the initiation and progression of numerous malignancies $(6,8,9,12,13,15)$. Dysregulation of SMURF1 is a common event in various types of cancer including PC $(8,9)$, breast (6,7) and ovarian cancer (13), and CRC (12). To the best of our knowledge, few studies have focused on the clinical values and the role of SMURF1 in GC to date. Thus, further studies concerning SMURF1 in GC may be helpful in the treatment of this deadly disease. The present study demonstrated the pattern of SMURF1 expression in the carcinogenesis of GC and its potential clinical significance in GC patients, and further investigated its effect and potential mechanisms in GC cells. The present study identified SMURF1 as a promising clinical biomarker of GC patients. The expression of SMURF1 in the GC tissues was notably increased compared to the tumor-adjacent specimens, and its increase was correlated with large tumor size, more lymph node metastasis and distant metastasis, as well as advanced TNM stage and decreased survival. Thus, SMURF1 potentially functions as a clinical marker in GC.

Then, we explored the biology of SMURF1 in GC, and demonstrated that silencing of SMURF1 expression inhibited cell proliferation and mobility in vitro and in vivo. In turn, SMURF1 overexpression significantly promoted cell proliferation as well as migration and invasion in GC cells. The prominent ability of SMURF1 to promote tumorigenesis reveals that it plays an oncogenic role in GC. Therefore, targeting SMURF1 may represent a favorable therapeutic strategy for GC treatment. Next, we explored a potential target gene that may be involved in the role of SMURF1. DAB2IP, a tumor-suppressor, plays a critical role in cancer cell growth and metastasis as well as other aspects during tumor progression (17). Epigenetic silencing is the main cause of dysregulated expression of DAB2IP in human cancers (20). The expression of DAB2IP is silenced by promoter methylation and histone modification in prostate cancer $(21,22)$. In bladder cancer, DAB2IP expression was suppressed by post-transcriptional regulation of miR-92b (23). In the present study, we revealed that silencing of SMURF1 upregulated DAB2IP expression while SMURF1 overexpression decreased DAB2IP expression in GC cells, suggesting that SMURF1 is a novel negative regulator of DAB2IP as previously reported (15). Notably, DAB2IP restoration resulted in similar effects to SMURF1 silencing in GC cells with weakened proliferation, migration and invasion. Thus, we support a preliminary theory that SMURF1 inversely regulates DAP2IP expression, resulting in the induction of growth and metastasis in GC. The PI3K/Akt pathway has been implicated in the growth and metastasis of GC $(24,25)$. In addition, the downstream targets of the PI3K/Akt pathway such as c-Myc and ZEB1 regulate proliferation, migration and invasion in GC cells $(18,19)$. In the present study, we reported that SMURF1 promoted the activation of PI3K/Akt and its downstream targets including c-Myc and ZEB1 via inhibition of DAB2IP in GC cells.

In conclusion, we revealed that SMURF1 as an oncogene is important in GC. Firstly, our results demonstrated that SMURF1 expression was increased in GC cell lines and tissues. Then, our clinical data revealed that SMURF1 may be used as a novel biomarker for GC. Moreover, SMURF1 overexpression resulted in enhanced proliferation and mobility possibly by DAB2IP inhibition in GC cells. Collectively, our results ascertained that SMURF1 may serve as a potential target in cancer therapeutics of GC.

\section{Acknowledgements}

The authors thank all the patients who participated in the present study.

\section{References}

1. Wang J, Yu JC, Kang WM and Ma ZQ: Treatment strategy for early gastric cancer. Surg Oncol 21: 119-123, 2012.

2. Marqués-Lespier JM, González-Pons M and Cruz-Correa M: Current perspectives on gastric cancer. Gastroenterol Clin North Am 45: 413-428, 2016.

3. Thrumurthy SG, Chaudry MA, Chau I and Allum W: Does surgery have a role in managing incurable gastric cancer? Nat Rev Clin Oncol 12: 676-682, 2015.

4. McLean MH and El-Omar EM: Genetics of gastric cancer. Nat Rev Gastroenterol Hepatol 11: 664-674, 2014.

5. Cao Y and Zhang L: A Smurf1 tale: Function and regulation of an ubiquitin ligase in multiple cellular networks. Cell Mol Life Sci 70: 2305-2317, 2013 .

6. Kwon A, Lee HL, Woo KM, Ryoo HM and Baek JH: SMURF1 plays a role in EGF-induced breast cancer cell migration and invasion. Mol Cells 36: 548-555, 2013.

7. Wang X, Jin C, Tang Y, Tang LY and Zhang YE: Ubiquitination of tumor necrosis factor receptor-associated factor 4 (TRAF4) by Smad ubiquitination regulatory factor 1 (Smurf1) regulates motility of breast epithelial and cancer cells. J Biol Chem 288: 21784-21792, 2013.

8. Suzuki A, Shibata T, Shimada Y, Murakami Y, Horii A, Shiratori K, Hirohashi S, Inazawa J and Imoto I: Identification of SMURF1 as a possible target for 7q21.3-22.1 amplification detected in a pancreatic cancer cell line by in-house array-based comparative genomic hybridization. Cancer Sci 99: 986-994, 2008.

9. Kwei KA, Shain AH, Bair R, Montgomery K, Karikari CA, van de Rijn M, Hidalgo M, Maitra A, Bashyam MD and Pollack JR: SMURF1 amplification promotes invasiveness in pancreatic cancer. PLoS One 6: e23924, 2011.

10. Xie P, Zhang M, He S, Lu K, Chen Y, Xing G, Lu Y, Liu P, Li Y, Wang S, et al: The covalent modifier Nedd8 is critical for the activation of Smurf1 ubiquitin ligase in tumorigenesis. Nat Commun 5: 3733, 2014.

11. Liu C, Billadeau DD, Abdelhakim H, Leof E, Kaibuchi K, Bernabeu C, Bloom GS, Yang L, Boardman L, Shah VH, et al: IQGAP1 suppresses T $\beta$ RII-mediated myofibroblastic activation and metastatic growth in liver. J Clin Invest 123: 1138-1156, 2013.

12. Nie J, Liu L, Xing G, Zhang M, Wei R, Guo M, Li X, Xie P, Li L, He F, et al: CKIP-1 acts as a colonic tumor suppressor by repressing oncogenic Smurf1 synthesis and promoting Smurf1 autodegradation. Oncogene 33: 3677-3687, 2014.

13. Wang W, Ren F, Wu Q, Jiang D, Li H, Peng Z, Wang J and Shi H: MicroRNA-497 inhibition of ovarian cancer cell migration and invasion through targeting of SMAD specific E3 ubiquitin protein ligase 1. Biochem Biophys Res Commun 449: 432-437, 2014.

14. Johansson E, Zhai Q, Zeng ZJ, Yoshida T and Funa K: Nuclear receptor TLX inhibits TGF- $\beta$ signaling in glioblastoma. Exp Cell Res 343: 118-125, 2016.

15. Li X, Dai X, Wan L, Inuzuka H, Sun L and North BJ: Smurf1 regulation of DAB2IP controls cell proliferation and migration. Oncotarget 7: 26057-26069, 2016.

16. Mendonsa AM, VanSaun MN, Ustione A, Piston DW, Fingleton BM and Gorden DL: Host and tumor derived MMP13 regulate extravasation and establishment of colorectal metastases in the liver. Mol Cancer 14: 49, 2015.

17. Liu L, Xu C, Hsieh JT, Gong J and Xie D: DAB2IP in cancer. Oncotarget 7: 3766-3776, 2016. 
18. Liu SQ, Yu JP, Yu HG, Lv P and Chen HL: Activation of Akt and ERK signalling pathways induced by etoposide confer chemoresistance in gastric cancer cells. Dig Liver Dis 38: 310-318, 2006

19. Yuan D, Xia H, Zhang Y, Chen L, Leng W, Chen T, Chen Q, Tang Q, Mo X, Liu M, et al: P-Akt/miR-200 signaling regulates epithelial-mesenchymal transition, migration and invasion in circulating gastric tumor cells. Int J Oncol 45: 2430-2438, 2014.

20. Tsai YS, Lai CL, Lai CH, Chang KH, Wu K, Tseng SF, Fazli L, Gleave M, Xiao G, Gandee L, et al: The role of homeostatic regulation between tumor suppressor DAB2IP and oncogenic Skp2 in prostate cancer growth. Oncotarget 5: 6425-6436, 2014.

21. Chen H, Toyooka S, Gazdar AF and Hsieh JT: Epigenetic regulation of a novel tumor suppressor gene $(h D A B 2 I P)$ in prostate cancer cell lines. J Biol Chem 278: 3121-3130, 2003.
22. Chen H, Tu SW and Hsieh JT: Down-regulation of human DAB2IP gene expression mediated by polycomb Ezh2 complex and histone deacetylase in prostate cancer. J Biol Chem 280: 22437-22444, 2005

23. Huang J, Wang B, Hui K, Zeng J, Fan J, Wang X, Hsieh JT, He D and $\mathrm{Wu} \mathrm{K}$ : miR-92b targets DAB2IP to promote EMT in bladder cancer migration and invasion. Oncol Rep 36: 1693-1701, 2016.

24. Xiong J, Li Z, Zhang Y, Li D, Zhang G, Luo X, Jie Z, Liu Y, Cao Y, Le Z, et al: PRL-3 promotes the peritoneal metastasis of gastric cancer through the PI3K/Akt signaling pathway by regulating PTEN. Oncol Rep 36: 1819-1828, 2016.

25. Shen X, Si Y, Wang Z, Wang J, Guo Y and Zhang X: Quercetin inhibits the growth of human gastric cancer stem cells by inducing mitochondrial-dependent apoptosis through the inhibition of PI3K/Akt signaling. Int J Mol Med 38: 619-626, 2016. 